\title{
Accentuate the (Gram) positive
}

\author{
Victor Nizet
}

Received: 19 January 2010 / Accepted: 19 January 2010 / Published online: 20 February 2010

(C) Springer-Verlag 2010

Keywords Gram-positive bacteria $\cdot$ Streptococcus . Special issue

In the last two decades, Gram-positive bacteria have become the most common organisms associated with bacteremia and sepsis [1]. Moreover, clinically-significant resistance to each class of conventional antibiotics has been shown by at least one Gram-positive species, posing an additional public health challenge $[2,3]$.

In June 2009 in Stockholm, the 140th Nobel Symposium continued a traditional focus on scientific fields in which breakthroughs were occurring, and was dedicated to "Acute Infections Caused by Gram-Positive Bacteria". This lively forum, expertly chaired by Profs. Anna Norrby-Teglund of the Karolinska Institute and Gunnar Lindahl of Lund University, brought together renowned investigators from more than a dozen countries to present cutting edge investigations on the virulence mechanisms of the leading Gram-positive bacterial pathogens and their interactions with the human immune system. Many new collaboration were found and audiences of scientists, both young and established, were inspired to contemplate new opportunities for translational studies to address the ongoing medical challenges. This special issue of the Journal of Molecular Medicine presents eight invited topical reviews from featured speakers of the 140th Nobel Symposium to further

V. Nizet $(\bowtie)$

Department of Pediatrics and Skaggs School of Pharmacy and Pharmaceutical Sciences, University of California San Diego, La Jolla, CA 92093, USA

e-mail: vnizet@ucsd.edu showcase this exciting area of contemporary human infectious disease research.

Streptococcus pneumoniae (SPN) is a leading agent of pneumonia, meningitis and sepsis throughout the world, affecting primarily those individuals at either extreme of the age spectrum. Recent analysis of World Health Organization data indicate that SPN is responsible for $11 \%$ of all deaths in children from 1 month to 5 years of age [4]. Coincidently, SPN has one of the most cherished histories in molecular biology research, being the experimental model in which Frederick Griffith demonstrated the principle of transformation [5] and in which Oswald Avery and colleagues demonstrated that DNA was the genetic material [6].

Like all the leading Gram-positive bacterial pathogens of humans, SPN commonly colonizes the human host without causing symptoms (carrier state) or generates milder, selflimited mucosal infections. The question of why bugs so innocent to most, can produce life-threatening invasive infections in a few, remains something of guiding paradox for researchers in the Gram-positive field. Jeffrey Weiser of the University of Pennsylvania proposes that many of the virulence factors promoting invasive SPN infection themselves provide adaptive advantages during the commensal state, either by meeting metabolic demands, resisting local mucosal immune responses, or allowing SPN to outcompete other members of the local flora [7]. The mechanisms of SPN invasion to produce lower respiratory tract, pneumonia, and meningitis are studied in the laboratory of Elaine Tuomanen of St. Jude Children's Research Hospital. She describes a common pattern of receptor-ligand interactions that facilitate penetration of host tissue barriers, such as binding of the laminin receptor by SPN adhesin $\mathrm{CbpA}$ and binding of the platelet activating factor receptor by SPN surface phosphorylcholine [8]. However, the cell 
signaling responses to SPN invasion differ markedly among host tissues, producing minimal damage to lung structure but irreversible injury to neurons and cardiomyocytes [8].

Staphylococcus aureus is recognized as a preeminent agent of serious bacterial disease, capable of producing infection in any organ and in any age group. Methicillinresistant strains of $S$. aureus (MRSA), once restricted to hospital settings, are now identified with alarming frequency in the general population, with several strains epidemic in communities across the USA. The Centers for Disease Control calculated that $>90,000$ Americans developed invasive methicillin-resistant S. aureus (MRSA) in 2005 and that $20 \%$ of these patients died in hospital from these devastating infections, a mortality incidence that may exceed that attributable to HIV/AIDS in the USA [9]. Frank DeLeo and colleagues at the National Institutes of Health Rocky Mountain Laboratories have traced the marked virulence potential of the prevalent community-acquired MRSA clones to a coordinate assemblage of innate immune resistance factors that promote survival against neutrophil clearance [10]. While MRSA are phagocytosed by host neutrophils, they utilize the potent cytotoxin alpha-hemolysin and the newly discoverd peptides known as phenol soluble modulins to cause rapid neutrophil lysis, leading to bacterial proliferation and further inflammatory tissue damage [10].

Innovative research probing interactions of Grampositive pathogens with host innate immune molecules, the coagulation system, and the microvascular endothelium has shed new light on invasive disease pathogenesis and clinical manifestions. Suzan Rooijakkers and colleagues at Utrecht University describe how $S$. aureus and another prominent invasive Gram-positive pathogen, group A Streptococcus (GAS) deploy a wide arsenal of secreted peptides and proteases that impair many of the key steps in complement recognition and opsonization [11]. Exciting data suggests these very complement evasion molecules could be co-opted for use as anti-inflammatory agents in human medicine [11]. Heiko Herwald at Lund University has been studying activation of the kallikein/kinin or contact system by $S$. aureus and GAS toxins and its role in the severe manifestations of sepsis such as hypotension and vascular leakage. From these data, it is suggested that blockage of kinin receptors or administration of $\mathrm{C} 1$ inhibitor, itself a regulator of the contact system, could have beneficial effects on mortality and lung injury induced by Gram-positive bacterial infection [12]. Profound alterations in the microcirculation, a byproduct of localized leukocyte adhesion and activation coupled to the systemic inflammatory response, are also manifested in Grampositive bacterial sepsis. Can Ince of the University of Rotterdam details how bacterial toxins including SPN pneumolysin, S. aureus superantigens or alpha-toxin, and GAS M protein or streptolysin O modulate leukocyte and platelet interactions with endothelium to promote microvascular dysfunction [13], a topic ripe for focused clinical research in the intensive care setting.

Development of effective vaccine prevention strategies is widely recognized as the ultimate strategy to alleviate the enormous global public health burden exacted by the leading Gram-positive bacterial pathogens. While the burden of disease caused by the most prevalent SPN serotypes has been diminished significantly in developed countries administering conjugate vaccines, these are not widely available in developing countries. Moreover, experimental vaccines against $S$. aureus, GAS, or group B Streptococcus are only in the early stages of clinical development. Richard Malley of Harvard Medical School presents some provocative new data to indicate protection against SPN colonization is not only dependent upon anti-capsular antibodies but also upon the presence of CD4+ IL-17A-producing T lymphocytes that recognize pneumococcal antigens [14]. Future vaccine studies, whether utilizing whole cells or purified antigen combinations, may wisely explore adjuvants and administration protocols that maximize both antibody and CD4+ IL-17A $T$ cell memory responses. Reverse vaccinology, which utilizes genome sequence information coupled to advanced tools of experimental proteomics, promises an expedited approach to identified highly-conserved, surface-expressed, and immunogenic protein antigens for Gram-positive bacterial vaccines. John Telford of Novartis Vaccines reviews the state-of-the-art in novel genome-based approaches to vaccine development [15].

With the leading Gram-positive bacterial pathogens, point-of-care clinicians and infectious disease specialists face formidable clinical challenge. Increasingly, they are encountering patients with severe, rapidly progressive, and difficult to treat infections, including those without previous underlying medical conditions. Fortunately, greater attention among microbiology and immunology researchers is being focused on Gram-positive pathogen-host interactions, yielding a wave of new research discoveries. By elucidating Gram-positive virulence factors and the host defenses they exploit, potential adjunctive approaches to therapy are being revealed. These novel treatments envision targeted neutralization of bacterial virulence phenotypes or pharmacologic augmentation of host innate immune defenses, and could be used in conjunction with classical antibiotic or vaccine strategies [16]. This special issue of the Journal of Molecular Medicine invites the broader academic medical community to likewise "think (Gram) positive".

\section{References}

1. Hodgkin KE, Moss M (2008) The epidemiology of sepsis. Curr Pharm Des 14:1833-1839 
2. Woodford N, Livermore DM (2009) Infections caused by Grampositive bacteria: a review of the global challenge. J Infect 59 (Suppl 1):S4-S16

3. Koomanachai P, Crandon JL, Nicolau DP (2009) Newer developments in the treatment of Gram-positive infections. Expert Opin Pharmacother 10:2829-2843

4. O'Brien KL, Wolfson LJ, Watt JP, Henkle E, Deloria-Knoll M, McCall N, Lee E, Mulholland K, Levine OS, Cherian T, Hib and Pneumococcal Global Burden of Disease Study Team (2009) Burden of disease caused by Streptococcus pneumoniae in children younger than 5 years: global estimates. Lancet 374:893-902

5. Griffith F (1928) The significance of pneumococcal types. J Hyg 27:113-159

6. Avery O, MacLeod C, McCarty M (1944) Studies on the chemical nature of the substance inducing transformation of pneumococcal types. Inductions of transformation by a desoxyribonucleic acid fraction isolated from pneumococcus type III. J Exp Med 79:137158

7. Weiser J (2010) The Pneumococcus: why a commensal misbehaves. J Mol Med 88(2) doi:10.1007/s00109-009-0557-x

8. Thornton JA, Durick-Eder K, Tuomanen EI (2010) Pneumococcal pathogenesis: 'innate invasion' yet organ specific damage. J Mol Med 88(2); (in press)

9. Klevens RM, Morrison MA, Nadle J, Petit S, Gershman K, Ray S, Harrison LH, Lynfield R, Dumyati G, Townes JM, Craig AS, Zell
ER, Fosheim GE, McDougal LK, Carey RB, Fridkin SK (2007) Invasive methicillin-resistant Staphylococcus aureus infections in the United States. JAMA 298:1763-1761

10. Graves SF, Kobayashi SD, DeLeo FR. (2010) Communityassociated methicillin-resistant Staphylococcus aureus immune evasion and virulence. J Mol Med 88(2) doi:10.1007/s00109-0090573-x

11. Laarman A, Milder F, van Strijp J, Rooijakkers S. (2010) Complement inhibition by gram-positive pathogens: molecular mechanisms and therapeutic implications. J Mol Med 88(2) doi:10.1007/s00109-009-0572-y

12. Oehmcke S, Herwald H. (2010) Contact system activation in severe infectious disease. J Mol Med 88(2). doi:10.1007/s00109009-0564-y

13. Legrand M, Klijn E, Payen D, Ince C. (2010) The response of the host microcirculation to bacterial sepsis: does the pathogen matter? J Mol Med 88(2); (in press)

14. Malley R (2010) Antibody and cell-mediated immunity to Streptococcus pneumoniae: implications for vaccine development. J Mol Med 88(2). doi:10.1007/s00109-009-0579-4

15. Mora M, Telford JL (2010) Genome-based approaches to vaccine development. J Mol Med 88(2). doi:10.1007/s00109-009-0574-9

16. Nizet V (2007) Understanding how leading bacterial pathogens subvert innate immunity to reveal novel therapeutic targets. J Allergy Clin Immunol 120:13-22 